Preface

\title{
Extrahemostatic Functions of Platelets and Coagulation Factors
}

\author{
Roger J. S. Preston, $\mathrm{PhD}^{1}$ Ton Lisman, $\mathrm{PhD}^{2}$ \\ ${ }^{1}$ Department of Molecular and Cellular Therapeutics, Irish Centre for \\ Vascular Biology, Royal College of Surgeons in Ireland, Dublin, \\ Ireland \\ 2 Section of Hepatobiliary Surgery and Liver Transplantation, \\ Surgical Research Laboratory, Department of Surgery, University of \\ Groningen, University Medical Center Groningen, Groningen, \\ The Netherlands
}

Semin Thromb Hemost 2018;44:89-90.

Vascular injury prompts the co-ordinated action of platelets and coagulation proteins to stem blood loss by thrombus generation. In response, the endothelium, in concert with anticoagulant plasma proteins, acts to control thrombus growth and prevent development of occlusive thrombi once the hemostatic process is initiated. ${ }^{1}$

In addition to these established roles, it is now apparent that an ever-increasing number of the cellular and plasma components required to either enable or regulate thrombus development also possess important physiological properties distinct from their traditional roles that, when dysregulated, can contribute to disease pathophysiology. 2,3

In this issue of Seminars in Thrombosis and Hemostasis, a selection of the diverse roles played by platelets and plasma hemostatic proteins in nonhemostatic physiology are reviewed. This issue is particularly timely given the significant recent progress made in better understanding these activities. The growing appreciation of their contribution to cancer progression, sepsis, chronic inflammatory disease, and cardiovascular disease, among others, is discussed further in this issue of the journal. Nevertheless, the pleiotropic activities of platelets and plasma coagulation proteins beyond their role in hemostasis remain incompletely defined, and efforts to understand these properties remain a rich vein of ongoing research. The current state-of-the-art understanding in several important areas is described herein.

The first part of this issue focuses on novel, nonhemostatic roles for platelets, where new insights in our understanding of how platelets can impact beyond their traditional role in clot formation continue to emerge. First, Kim et al outline newly identified roles for platelets in shaping innate and adaptive immune responses to infection and provide examples of how uncontrolled platelet activation can tip the balance toward unfavorable immune activation and creation of an inflammatory milieu. ${ }^{4}$ On a related theme, Maiocchi et al then detail how platelets contribute to the development of a local "thromboinflammatory" environment upon ischemic-reperfusion injury that commonly arises after stroke or acute myocardial infarction. $^{5}$ Of particular interest, the authors consider how the constitutively elevated thromboinflammatory properties of platelets from diabetic patients may contribute to their significantly increased risk of cardiovascular disease. Lisman and Luyendyk then discuss the clinical and experimental evidence underlying the complex roles played by platelets in progression of liver disease, a complex disorder well established to significantly impact upon global hemostasis. In addition, they review the current evidence for a potential role for platelets in driving liver regeneration after injury. ${ }^{6} \mathrm{Next}$, Suzuki-Inoue et al describe how platelet C-type lectin-like receptor 2 (CLEC-2)podoplanin signaling plays a crucial role in blood vessel formation, wound healing, and maintenance of vascular integrity during development. Furthermore, they describe the deleterious outcomes associated with CLEC-2 signaling and its contribution to tumor metastasis. ${ }^{7}$ Tjon-Kon-Fat et al then consider the role played by platelets in cancer cell biology, either by activated platelet release of proinflammatory cytokines and chemokines or via direct tumor cell interactions. In particular, they discuss the remarkable phenomenon of "tumor-mediated platelet education," whereby platelet nucleic acid uptake enables ingestion of tumor-specific RNA transcripts that can subsequently impact platelet behavior and activity. ${ }^{8}$ Interestingly, these tumor-mediated modifications in platelet RNA content can be correlated with specific malignant disease profiles, highlighting the potential utility of platelet
Address for correspondence Roger J. S. Preston, PhD, Department of Molecular and Cellular Therapeutics, Irish Centre for Vascular Biology, Royal College of Surgeons in Ireland, 123 St Stephen's Green, Dublin, Ireland

(e-mail: rogerpreston@rcsi.ie).
Issue Theme Extrahemostatic Functions of Platelets and Coagulation Factors; Guest Editors: Roger J. S. Preston, PhD, and Ton Lisman, PhD.
Copyright $\odot 2018$ by Thieme Medical Publishers, Inc., 333 Seventh Avenue, New York, NY 10001, USA. Tel: +1(212) 584-4662.
DOI https://doi.org/ 10.1055/s-0037-1612624. ISSN 0094-6176. 
RNA analysis as a future diagnostic approach that may enable individualized treatment approaches for cancer patients.

The second part of this issue focuses on how protein components of the hemostatic system exhibit important functions distinct from their traditional roles in either enabling or controlling clot formation. Tissue factor, well known for its essential role in initiation of hemostatic blood clot formation, also has diverse signaling properties when bound to distinct ligands and/or signaling coreceptors. ${ }^{9}$ The review by Hoffman in this issue considers a potentially important additional role for tissue factor beyond clot formation in the cellular response to tissue injury, by triggering signaling pathways leading to wound healing, angiogenesis, and tissue regeneration..$^{10}$ Zeerleder subsequently provides an important overview on factor VII-activating protease (FSAP) biology, a plasma protein whose challenging physical properties and multiple substrates have hindered a complete understanding of its physiological purpose. ${ }^{11}$ In this review, the author describes the current understanding of the role of FSAP in hemostasis and demonstrates its potential role in both acute and chronic inflammatory disease states. Next, O'Sullivan et al describe the potential role of von Willebrand factor (VWF) in tumor metastasis and cancer. ${ }^{12}$ VWF is crucial for normal hemostasis and deficient VWF expression or function leads to von Willebrand disease, the most common inherited bleeding disorder. Although the adhesive properties of VWF are normally considered in the context of high-affinity FVIII binding and platelet adhesion, there exist an increasing number of experimental and clinical studies that link VWF binding of established and novel ligands to the development of vascular dysfunction. In this review, O'Sullivan et al collate evidence from recent studies that implicate an intriguing role for VWF in binding to tumor cells to modulate their behavior and migration.

The final part of this issue considers the extrahemostatic activity of endogenous anticoagulant pathways and our current understanding of their dual role in controlling both coagulation and inflammation. The protein $C$ pathway is particularly important in this respect, and the cytoprotective properties of activated protein C (APC) prompted previous development of its recombinant form for the treatment of severe sepsis. ${ }^{13}$ Furthermore, the use of a recombinant nonanticoagulant APC variant to mitigate tissue-type plasminogen activator neurotoxicity in ischemic stroke patients is currently being trialled. ${ }^{14}$ Despite growing evidence for the importance of its immunomodulatory activity, the breadth of the APC cell signaling repertoire and its usage to control innate and adaptive immunity remains incompletely understood and this topic is reviewed by McDonnell et al. ${ }^{15}$ Moreover, protein S, a crucial anticoagulant protein that augments APC and tissue factor pathway inhibitor anticoagulant activity, has been shown to also exhibit potent signaling activities distinct from its anticoagulant partners. These properties are mediated by binding to and signaling via the Tyro3/Axl/Mer signaling receptor family. ${ }^{16,17}$ Furthermore, protein $S$ plays additional important roles in apoptotic cell phagocytosis and complement regulation. In this issue,
Dahlback provides an overview of the multiple anti-inflammatory and immunoregulatory properties of protein $S .^{18}$

In summary, the reviews featured in this issue of the journal provide a snapshot of how components of the hemostatic system act outwith their canonical role in either driving clot formation or in regulating clot growth. Furthermore, they underscore the pathophysiological consequences when these distinct properties become dysregulated, particularly in the development of inflammatory and malignant disease. More optimistically, these insights also highlight the potential diagnostic and therapeutic opportunities provided by a fuller understanding of the extrahemostatic properties of platelets and anticoagulant proteins.

\section{References}

1 Furie B, Furie BC. Mechanisms of thrombus formation. N Engl J Med 2008;359(09):938-949

2 Esmon CT. The interactions between inflammation and coagulation. Br J Haematol 2005;131(04):417-430

3 Morrell CN, Aggrey AA, Chapman LM, Modjeski KL. Emerging roles for platelets as immune and inflammatory cells. Blood 2014;123 (18):2759-2767

4 Kim SJ, Davis RP, Jenne CN. Platelets as modulators of inflammation. Semin Thromb Hemost 2018;44(02):91-101

5 Maiocchi S, Alwis I, Wu M, Yuan Y, Jackson SP. Thrombo-inflammatory functions of platelets in ischemia-reperfusion injury and its dysregulation in diabetes. Semin Thromb Hemost 2018;44 (02):102-113

6 Lisman T, Luyendyk JP. Platelets as modulators of liver diseases. Semin Thromb Hemost 2018;44(02):114-125

7 Suzuki-Inoue K, Tsukiji N, Shirai T, et al. Platelet CLEC-2-roles beyond hemostasis. Semin Thromb Hemost 2018;44(02):126-134

8 Tjon-Kon-Fat LA, Sol N, Wurdinger T, Nilsson RJA. Platelet RNA in cancer diagnostics. Semin Thromb Hemost 2018;44(02):135-141

9 Versteeg HH, Ruf W. Emerging insights in tissue factor-dependent signaling events. Semin Thromb Hemost 2006;32(01):24-32

10 Hoffman M. The tissue factor pathway and wound healing. Semin Thromb Hemost 2018;44(02):142-150

11 Zeerleder S. Factor VII activating protease - hemostatic protein or immune regulator? Semin Thromb Hemost 2018;44(02):151-158

12 O'Sullivan JM, Preston RJS, Robson T, O'Donnell JS. Emerging roles for von Willebrand factor in cancer cell biology. Semin Thromb Hemost 2018;44(02):159-166

13 Bernard GR, Vincent JL, Laterre PF, et al; Recombinant human protein C Worldwide Evaluation in Severe Sepsis (PROWESS) study group. Efficacy and safety of recombinant human activated protein C for severe sepsis. N Engl J Med 2001;344(10):699-709

14 Griffin JH, Fernández JA, Lyden PD, Zlokovic BV. Activated protein $C$ promotes neuroprotection: mechanisms and translation to the clinic. Thromb Res 2016;141(Suppl 2):S62-S64

15 McDonnell CJ, Soule EE, Walsh PT, O'Donnell JS, Preston RJS. The immunoregulatory activities of activated protein $C$ in inflammatory disease. Semin Thromb Hemost 2018;44(02):167-175

16 Carrera Silva EA, Chan PY, Joannas L, et al. T cell-derived protein S engages TAM receptor signaling in dendritic cells to control the magnitude of the immune response. Immunity 2013;39(01): 160-170

17 Rothlin CV, Ghosh S, Zuniga EI, Oldstone MB, Lemke G. TAM receptors are pleiotropic inhibitors of the innate immune response. Cell 2007;131(06):1124-1136

18 Dahlback B. Vitamin K-dependent protein S: beyond the protein C pathway. Semin Thromb Hemost 2018;44(02):176-184 